\title{
THE THERMO-STRESSED STATE OF STEAM TURBINE ROTORS DURING PLANT START-UP
}

\author{
V. GOLOSHUMOVA \& YU. BRODOV \\ Ural Federal University, Russia.
}

\begin{abstract}
This article suggests the use of the value of the thermo-stressed state in the turbine steam input stage as one of the key parameters characterizing the start-up reliability of the turbine plant cycle. The authors present the principles of the development of the steam turbine rotor warming model for continuous monitoring of its thermo-stressed state, using a personal computer in real time. The research conducted on the thermo-stressed state of the rotor using a universal CAE programme allowed the hypothesizing of the maximum thermo-stressed states as can be calculated with sufficient precision by 'characteristic' temperature differences inside the rotor. It is proposed to evaluate the thermo-stressed states using regression dependence on the 'characteristic' differences of the real-time temperature field. Samples for the regression analysis are obtained from preliminary results of CAE programme calculations. An example is given for the whole range of the necessary computational research to produce modules of a control device for the steam turbine T-110/120-130. The modules are tested in the MATLAB Simulink environment.

Keywords: automation, finite element method, model, modelling, personal computer, real time, steam turbine rotor, thermo-stressed state.
\end{abstract}

\section{INTRODUCTION}

The activities focusing on full or partial automation of the transient modes of steam turbine plants are known to be one of the most crucial areas aimed at improving thermal power plants. Automation reduces the impact of human factors including the lack of experienced staff, by utilizing these optimal transient modes; it also provides a number of other advantages. First, the reliability of the main equipment improves and its lifetime increases. Secondly, there are energy savings due to a reduction in the duration of the start-up time. Thirdly, it is possible to collect statistical information to forecast the state of metal fatigue of the main equipment; this, in its turn, allows for the efficient and timely planning of repair activities. The authors suggest taking the level of the thermo-stressed state in the control partial stage input section of steam turbines as one of the key parameters characterizing the reliability of the plant start-up process.

A number of companies and organizations in Russia are now working on the integrated automation of power facilities. The most common software systems developed are SPPA T3000 (Siemens, JSC Interavtomatika ZAO), KVINT (JSC NIITeplopribor OAO), Sargon (JSC HBT-Avtomatika ZAO), Tornado (JSC MST ZAO), and Mentor (JSC NPO TsKTI $\mathrm{OAO}$ ). These systems use cutting-edge information technologies having a modular structure to cover a large part of the main and supplementary equipment of the steam plant. To automate the transition modes of operation of sophisticated machinery such as steam turbines, however, algorithms and techniques developed mainly in the 1980-1990s are utilized. These algorithms, in particular, include the use of temporary programmes: these in fact represent the turbine starting instructions in a particular programming language.

The main constraint associated with the automation of the transient modes of a powerful steam turbine is the need to evaluate the thermo-stressed state of the high- and mediumpressure rotors [1-5]. For example, a 'critical' element limiting turbine start-up speed for the 
T-110/120-130 (T-110) model [5] is the control partial stage of the high-pressure rotor; in this stage, it is technically very difficult to arrange direct temperature measuring of the rotor, and it is therefore necessary to use mathematical modelling.

\section{SIMULATION OF THE MULTI-TEMPERATURE FIELD IN ROTOR, USING A PERSONAL COMPUTER IN REAL TIME}

The authors propose an alternative approach to modelling the unsteady temperature field of the rotor on a limited capacity computer; this consists of using a specially optimized finite element model of the turbine start-up process in real time [2-5]. Figure 1 shows a diagram of the necessary steps in the process using the optimized model.

By the optimized model, it is meant the minimization of calculation resources (computer capacity and the amount of operational memory), which are necessary for the realization of this model maintaining the required accuracy. To do this, a master model must be created. It is also necessary to get a 'precise decision' of the rotor temperature field and its deflected mode with the help of CAE software on a high capacity computer. While creating the optimized model, a part of rotor with maximal temperature tension was chosen from the master model for calculations on the limited capacity computer, then the finite elements were reasonably enlarged and a simple model for rotor steel properties was set up. Temperature field calculations for the selected part coincided both in the master and the optimized models.

Using the finite element method (FEM) in heat conduction modelling permits accounting for the actual geometric shape of the rotor, the complex boundary conditions (BC) of heat transfer, and the temperature-dependent rotor material properties.

The preliminary optimization of the computational grid makes it possible to achieve an acceptable modelling rate for the process of the warm-up speed of the rotor on relatively low-power computers, while maintaining the required accuracy. FEMs are now known to be used in real time as computer graphics for the realistic visualization of objects in training facilities [6].

Figure 2 demonstrates a diagram of the high-pressure cylinder of a steam turbine T-110/120130 and a fragment of a 3.704-m model of the turbine T-110/120-130 high-pressure rotor temperature field at the 20th minute of a cold start, resulting from calculations with the general-purpose programme CAE. Having analysed the results, the simulation of the rotor thermo-stressed state during the cold start showed that for this turbine, relevant areas are the surface of the axial hole along the centre-line (under the disc of the massive double-crown control stage, the 'cold spot' effect) and the fillets control stage. It has been found that in the turbine start-up modes, the maximum temperature stress is observed not only in the heated

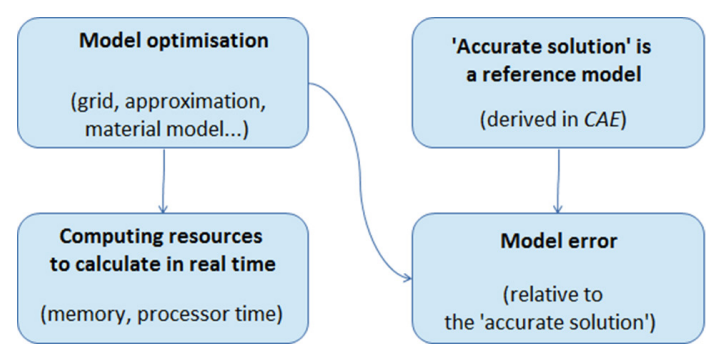

Figure 1: The diagram of the obligatory stages for calculations on the limited capacity computer. 


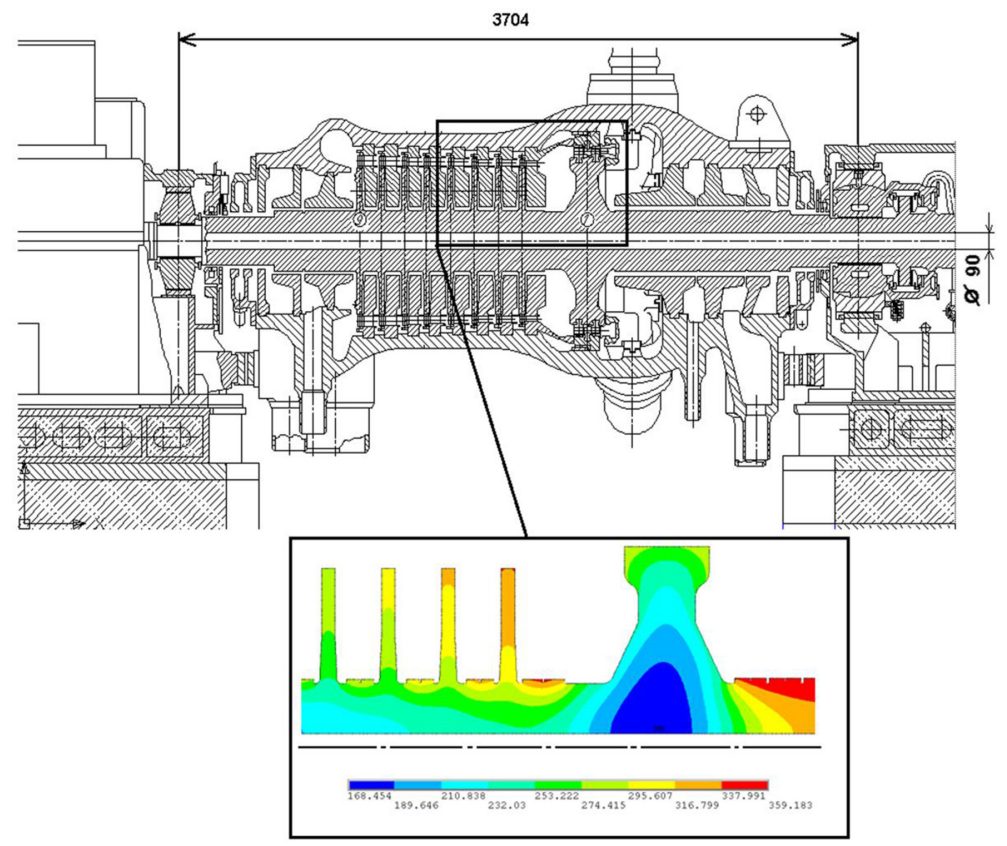

Figure 2: The diagram of the high-pressure cylinder of the steam turbine T-110/120-130 and a fragment of the rotor temperature field, obtained on the master model at the 20th minute of a cold start.

bore but also in the inner bore under the control stage disc (a tensile tangential stress of $\sigma_{\max }=280 \mathrm{MPa}$ ). It should be noted that the zone of maximal temperature stresses coincides with the zone of stresses caused by centrifugal forces.

There are many finite element packages, such as ANSYS and ABAQUS [2-7], available for conducting the transient thermal analysis. These packages allow the designer to vary the ambient temperature with time, to vary the convective heat transfer coefficients and heat flux with time/temperature, and also allow heat generation to be applied.

Figure 3 shows a geometric turbine T-110/120-130 high-pressure rotor optimized model during warm-up in the control stage. This geometric model, sized $0.620 \mathrm{~m}$, was selected for calculations on a computer with limited power.

The model developed by the authors is implemented in the MATLAB Simulink environment in the module library format.

The 'Loading curve for T-110' module produces the rotation rate variation graphs and power variation diagrams during start-up according to the instruction manual.

The 'Convection parameters for T-110' module converts the output signals of the previous module into the parameters for the calculation of the convective heat transfer. The model can be used in the automated process control system of a real turbine to generate the $\mathrm{BC}$ of full-time data from sensors (the parameters of live steam, turbine power, rotor speed, temperature in the chamber of the turbine control stage, and others).

$\mathrm{BC}$ are computed by 'BC collector for T-110'. The heat transfer coefficients from steam to the rotor metal at nominal operation of the turbine were calculated using the widely known Nusselt number correlations' equations [8]. To calculate the heat transfer on the cylindrical 

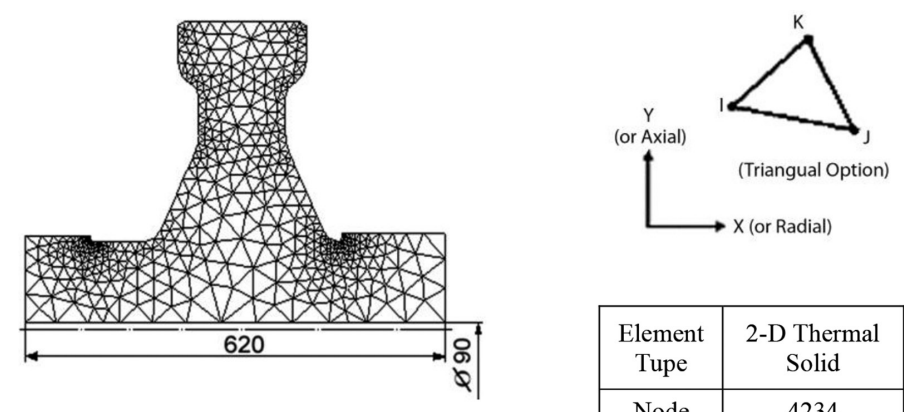

\begin{tabular}{|c|c|}
\hline $\begin{array}{c}\text { Element } \\
\text { Tupe }\end{array}$ & $\begin{array}{c}\text { 2-D Thermal } \\
\text { Solid }\end{array}$ \\
\hline Node & 4234 \\
\hline
\end{tabular}

Figure 3: Optimized model rotor cylinder high-pressure steam turbine T-110/120-130 for calculations on a limited capacity computer.

surface of the rotor in the control stage chamber, in the case of the dominant axial steam movement over the rotational $\operatorname{Re}_{z}>>\operatorname{Re}_{\varphi}$, the following equation was used:

$$
\mathrm{Nu}=0.02 \cdot \operatorname{Re}_{Z}^{0.8} \cdot \operatorname{Pr}^{0.33},
$$

where $\mathrm{Nu}=a \cdot d / k$ is the Nusselt number, $\operatorname{Re}=d \cdot w \cdot \rho / \mu$ is the Reynolds number, and $\operatorname{Pr}$ is the Prandtl number. In these latter equations, $\alpha$ is the flow convective heat transfer coefficient, $d$ is the characteristic length, $k$ is the thermal conductivity of steam, $w$ is the characteristic velocity of steam $\mu$ is the coefficient of dynamic viscosity of steam, and $\rho$ is the density of steam. Selection of the characteristic length should be in the direction of growth (or thickness) of the boundary layer. In the case of the dominant rotational steam movement over the axial flow $\operatorname{Re}_{\varphi}>>\operatorname{Re}_{Z}$, the following equation was used:

$$
\mathrm{Nu}=0.038 \cdot\left(\frac{\operatorname{Re}_{\varphi}}{2} \cdot \sqrt{\frac{R_{2}-R_{1}}{R_{1}}}\right)^{0.8} \cdot \operatorname{Pr}^{0.43}
$$

The determining size is the value $\left[2\left(R_{2}-R_{1}\right)\right]$, which is twice the stator-to-rotor gap. The values $\operatorname{Re}_{\varphi}$ and $\operatorname{Re}_{Z}$ are determined by circumferential and lateral velocities, respectively.

To calculate the heat transfer on the side surfaces of the control stage disc, the equation is

$$
\mathrm{Nu}=0.027 \cdot \operatorname{Re}^{0.8} \cdot \operatorname{Pr}^{0.6} \text {. }
$$

In both cases, the determining size $R$ is the average disc radius times the height; determined by the rate is the circumferential velocity at a given radius.

To evaluate the heating of the steam turbine rotor during start-up, heat transfer coefficients must be used in the calculation.

To calculate the rotor temperature field of the steam at normal loading, heat transfer coefficient is used:

$$
a_{0}=\mathrm{Nu}_{0} \cdot \frac{k_{0}}{d},
$$

where $\alpha_{0}$ is the heat transfer coefficient from steam to metal under nominal conditions, $\mathrm{Nu}_{0}$ is the Nusselt number under nominal conditions, $k_{0}=f\left(t_{0}, P_{0}\right)$ is the steam thermal 
conductivity, a function of the temperature and pressure under nominal conditions; the index ' 0 ' corresponds to the nominal steam parameters; $t$ is the temperature; and $P$ is the pressure.

At intermediate loads, coefficients were determined by the formula obtained after transformation of eqns (1)-(3), in general terms:

$$
\alpha=a_{0} \cdot \frac{k}{k_{0}} \cdot\left(\frac{G}{G_{0}} \cdot \frac{\mu_{0}}{\mu}\right)^{0.8} \cdot\left(\frac{\operatorname{Pr}}{\operatorname{Pr}_{0}}\right)^{l},
$$

where $\alpha$ is the heat transfer coefficients from steam to metal, $k=f(t, P)$ is the steam thermal conductivity, a function of the temperature and pressure, $G=f(N, n)$ in which $G$ is the mass steam flow; $\operatorname{Pr}=f(t, P)$ is the Prandtl number for steam; $l$ is the exponent in the Prandtl number given by formulas (1)-(3); $N$ is the power output turbine; and $n$ is the rotor speed.

The module 'Material model for P2MA' provides the thermo-physical properties of the rotor steel.

The 'Load Mesh' automatically downloads the finite element mesh from the prepared file immediately prior to simulation and calculations on a limited capacity personal computer.

The basic module of the model is the solver ('Discrete FEM solver'), which implements the heat conduction solution for the axially symmetric geometrical rotor model using the FEM in real time. The matrix equation for this problem is

$$
[C] \cdot\left\{\frac{\mathrm{d} T}{\mathrm{~d} t}\right\}+[K] \cdot\{T\}=\{F\},
$$

where $C$ is the specific heat matrix responsible for the temperature time dependence; $T$ is the temperature vector; $K$ is the conductivity matrix responsible for the material characteristics; $F$ is the load vector responsible for heating; and $t$ is the time.

Equation (6), a system of ordinary differential equations, is solved implicitly by the firstorder Euler method with the formula:

$$
T_{L+1}=T_{L}+\Delta t \cdot\left((1-\Theta) \cdot T_{L}+\Theta \cdot T_{L+1}\right),
$$

where index $L$ corresponds to the time increment number; $\Delta t$ is the time step; and $\Theta$ is the integration parameter. As a result, the temperatures at the nodes of the computational grid are obtained with the solver output.

The 'Plot Result' module provides visualization of the rotor temperature field.

\section{THERMO-STRESSED STATES OF THE TURBINE ROTOR IN THE CONTROL PARTIAL STAGE DURING START-UP}

In theory, the timeframe over which the turbine can start is limited by thermal stress-related constraints. These are directly connected to material properties and to radial temperature gradients to which the material is exposed. In the elastic material range, the thermal stress on a body can be expressed by equation:

$$
\sigma=\cdot A \cdot \Delta T
$$

where $A=E \cdot \beta_{m} /(1-v), E$ is Young's modulus; $\beta_{\mathrm{m}}$ is the mean linear expansion coefficient; $v$ is Poisson's ratio, the traverse expansion coefficient; and $\Delta T=T_{m}-T_{l}$, where $T_{m}$ is the mean final temperature and $T_{l}$ is the initial temperature. 
Additionally, an axial temperature gradient arises in the rotor. The authors took this fact into account.

In principle, FEM can also be used for stress computation but in this paper we used an alternative approach that does not require any significant computational resources.

The study conducted on the thermo-stressed state of the T-110/120-130 steam turbine rotor with the general-purpose CAE programme made it possible to hypothesize that the maximum thermo-stressed states can be calculated with sufficient accuracy from the "characteristic temperature differences' in the rotor body. Heuristic methods made it possible to choose 'characteristic temperature differences' in the radial and axial directions. Regression analysis confirmed the independence of the selected differences.

The maximum thermo-stressed states occur at different starting points in various parts of the rotor control partial stage. The simulation of the thermo-stressed state on the master model with the help of CAE software showed that for the turbine considered these areas are: the surface of the axial hole along the centre-line under the disc of the massive double-crown control stage (the 'cold spot' effect) and the fillets of the control stage.

The authors calculated the thermal stress during the turbine start-up based on regression formulas: they obtained statistical information to use in numerical calculations based on the master model. The regression interdependence of the thermal stresses and the selected temperature differences was obtained by the method of least squares. The interdependence of the thermo-stressed states in these stages, and the characteristic temperature difference, is expressed as follows:

$$
\sigma_{j}=A_{j 0}+\sum_{i} A_{j i} \cdot \Delta T_{i}, \quad j=1 \ldots J, \quad i=1 \ldots I
$$

where $J$ is the number of points in the control partial stage in which the thermo-stressed state is approximate; $\sigma_{j}$ is the thermo-stressed state; $I$ is the number of characteristic temperature differences; $\Delta T_{i}$ is the 'characteristic temperature difference'; and $A$ is the regression coefficient. The values of the characteristic differences are generated from the nodal temperatures in the 'Differences Extractor' module.

The computation of the thermo-stressed state is implemented in the 'Stress Approximation' module. If the thermo-stressed states exceed the specified limit of allowable stresses and then the yield strength of the rotor steel, the operator's terminal will receive a corresponding warning. Under the maximum permissible thermo-stressed state, the authors use the yield strength value of the rotor steel, taking into account a safety margin. With the safety margin at 1.6, the limit value of the thermo-stressed states in a controlled area of the high-pressure cylinder rotor will be $\left[\sigma_{\lim }\right] \leq \sigma_{0.2 \%} / 1.6=460 / 1.6 \approx 287.5 \mathrm{MPa}$.

The stresses of centrifugal forces at steam turbine rotor rotation are calculated based on the industrial Russian standard 'Steam stationary turbines. Calculations of discs and rotors strengths'.

While making the basic calculations based on the industrial standard, temperature stresses are not taken into account; however, mechanical material properties are accounted based on the assumption of distribution of rotor metal temperature. For the ratio of the reserve factor to yield strength $\sigma_{0.2 \%}$ the value 1.6 is taken.

\section{METHODOLOGY APPLICATION RESULTS}

The device for monitoring the thermo-stressed state of the T-110/120-130 steam turbine high-pressure rotor is implemented virtually in the Rede MATLAB Simulink programme. Figure 4 shows the developed modular diagram. When calculating the thermo-stressed state 


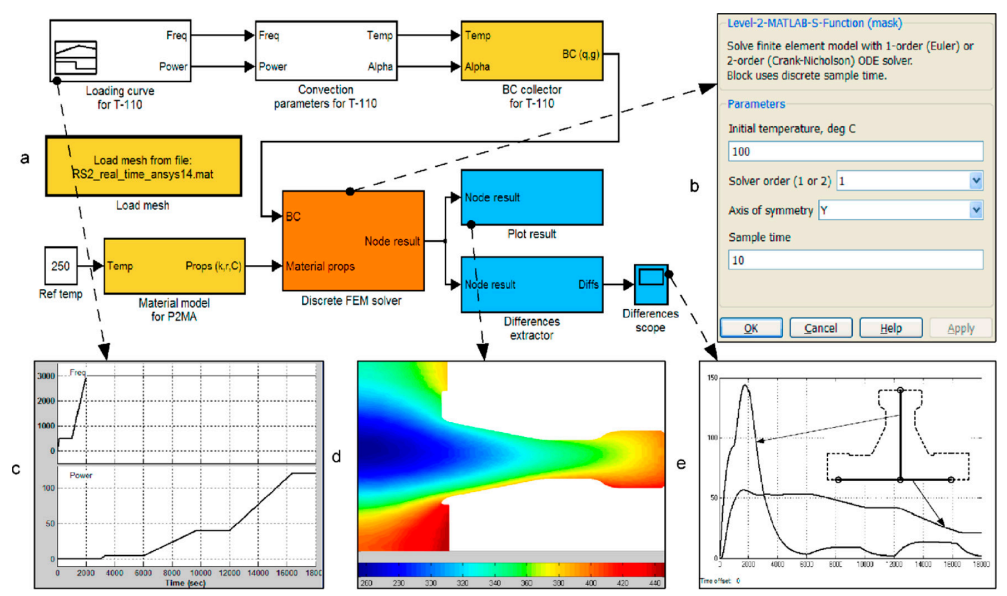

Figure 4: Steam turbine T-110/120-130 high-pressure rotor in the Matlab Simulink environment: modular diagram of the warm-up. (a) Block diagram of the model in the Simulink environment, (b) parameter window, finite element model's solver, (c) turbine loading schedules during start-up from cold state, (d) the temperature field of the rotor at the 35 min after 'kick-in', and (e) temperature differences at characteristic sections of the rotor body.

of the steam turbine rotor T-110/120-130 in real time on a personal computer (Intel Pentium 4, $2.8 \mathrm{GHz}, 2048 \mathrm{MB}$ RAM) in the MATLAB Simulink environment, the maximum time required for the calculation was $0.7 \mathrm{~s}$.

\section{CONCLUSION}

First, temperature field calculations should be done by the finite elements method on the optimized model of the rotor. Next, 'characteristic temperature differences' and temperature tensions may be calculated. This process does not require a high capacity computer.

As an example, the whole range of the computational research for the steam turbine $\mathrm{T}-110 / 120-130$ is presented: that is, the characteristic temperature differences on the rotor body uniquely determining the thermo-stressed states in the critical zone of the rotor, which is the control stage. The regression interdependence linking characteristic temperature differences with thermo-stressed states in the rotor was obtained.

It is necessary to introduce a new standard metal temperature measurement of the turbine rotor in the axial hole under the disc of the control stage for comparison of the temperature field based on the optimized model in time to start. The authors are aware that the set-up of the standard temperature measurement of the rotor requires a lot of workload on placing and taking out measuring thermocouples from the rotor axial hole.

\section{REFERENCES}

[1] Plotkin, E.R., Leizerovich, A.S. \& Berkowitz, Ya.D., Information support of the operator when starting the turbine 228 MW. Power Station, 10, pp. 14-21, 2013 [in Russian].

[2] Goloshumova, V.N., Brodov, Yu.M. \& Smirnov, A.A., Simulation of the temperature field of the rotor of the steam turbine control systems for its thermal stress state. Reliability and Energy Security, 4, pp. 59-64, 2013 [in Russian]. 
[3] Goloshumova, V.N., Smirnov, A.A. \& Brodov, Yu.M., Controlling the startup modes temperature steam turbine rotor condition. Heavy Engineering, 3, pp. 40-43, 2011 [in Russian].

[4] Smirnov, A.A., Goloshumova, V.N. \& Brodov, Yu.M., Simulation of temperature field of the rotor of a steam turbine in real time. Reliability and Energy Security, 2, pp. 30-36, 2012 [in Russian].

[5] Goloshumova, V.N., Brodov, Yu.M., Klyainrok, I.Yu. \& Smirnov, A.A., Controlling the startup modes of cogeneration steam turbines operating as part of combined - cycle power plants. Thermal Engineering, 12, pp. 1-9, 2012 [in Russian].

[6] Berkley, J., Turkiyyah, G., Berg, D., Ganter, M. \& Weghorst, S., Real-time finite element modeling for surgery simulation: an application to virtual suturing. IEEE Transactions Visualization and Computer Graphics, 10(3), pp. 314-325, 2004. http://dx.doi.org/10.1109/TVCG.2004.1272730

[7] Ehrsam, A., Schreier, T, Brunneur, P. \& Mitra, A., Steam turbine start-up optimization tool based on ABAQUS and python scripting, Simulia Customer Conference, London, 2009, pp. 1-8, available at http: www 3ds.com/products-services/simula/resourcecenter/by-inductry/.

[8] Leyzerovich, A.Sh., Steam turbines for modern fossil-fuel power plants, Fairmont Press, 2008, p. 550. 